\title{
Case report blunt trauma: an uncommon cause of bile duct injury
}

\begin{abstract}
Disruption of the biliary tree secondary to blunt trauma is a rare cause for extrahepatic bile duct injury.1-4 It seldom occurs alone, hence, this type of injury is easily overshadowed by more overt surgical emergencies and may go undiagnosed, leading to complications and potentially adverse outcomes in the later course. A variety of imaging modalities have been used to varying degrees of success in identifying biliary tree disruption for stable patients but in emergent settings exploratory laparotomy remains the most efficacious means for identifying injury $[4,5,6]$. The spectrum of severity ranges from severe ones such as transection or laceration, to contusion and hematoma. The incidence of bile leaks following hepatobiliary trauma ranges from 0.5 to $2.1 \%$ depending on the methods used to diagnose the bile leak. We present here a rare case with injury to both IHBR and EHBD as a consequence to abdominal trauma.
\end{abstract}

Keywords: undiagnosed, hepatobiliary, extrahepatic, IHBR, EHBD
Volume 5 Issue 2 - 2018

\section{Arpit Bandi, Kulwant Singh, Aashirwad Datey, Ravi Pratap Singh}

Department of General Surgery, Peoples College of Medical Sciences and Research Centre, India

Correspondence: Arpit Bandi, Department of General Surgery, Peoples College of Medical Sciences and Research Centre, Bhopal, M.P (4620I0), India, Tel +9I-8959644985, Email arpitbandi@gmail.com

Received: October II, 2017 | Published: March 16, 2018

\section{Case report}

A $25 \mathrm{yr}$ old male presented to the emergency at around 3am with an alleged history of road traffic accident due to motorcycle collision with a heavy vehicle. On presentation, Patient was hemodynamically stable. On Gross examination, He was conscious and oriented and had no signs of any head injury. He had mild pallor and no icterus or clubbing at the time of admission. He had multiple facial injuries with unstable mandibular arch along with a split maxilla. Also, there were multiple lacerated wounds intra-orally and extra-orally but no gross injury mark over the abdomen or other regions. On per abdomen examination, the abdomen was soft with no visible distension. There was no guarding or rigidity although the patient had mild tenderness in right hypochondrium. Apart from this, the chest and pelvic compression were negative.

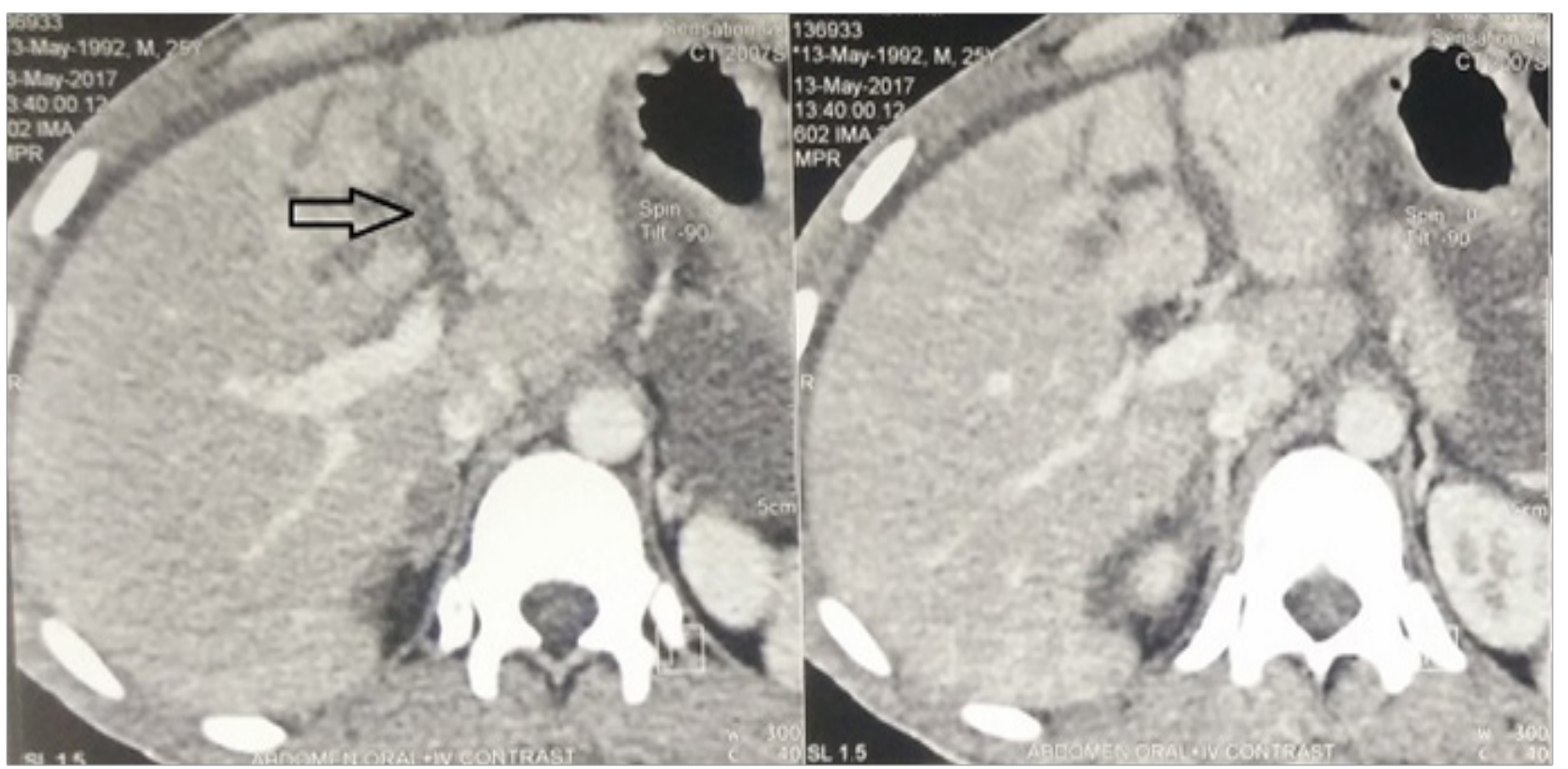

Figure I Initial CECT abdomen Showing lacerated 4A segment of liver (black arrow) around the left portal vein.

USG abdomen suggested a small hypoechoic lesion in left lobe of liver s/o liver contusion. CECT abdomen was done which revealed a Laceration in the segment $4 \mathrm{~A}$ of liver (around $4.6^{*} 3.45 \mathrm{~cm}$ ) around the left portal vein reaching up to the liver capsule with mild- moderate hemoperitoneum (Figure 1). Initial Blood investigations revealed Hemoglobin of $8 \mathrm{gm} / \mathrm{dl}$, Bilirubin $0.70 \mathrm{mg} / \mathrm{dl}$, raised SGOT180.3IU/L (range 5-40IU/L) and SGPT-189.4IU/L (range 5-40IU/L) and reduced Serum proteins (Total $-4.56 \mathrm{gm} / \mathrm{dl}$, range $6.0-8.0 \mathrm{gm} / \mathrm{dl}$, 
Albumin-2.81gm/dl, Globulin-1.75gm/dl). Patient was transfused 1 unit Packed Red blood cells and 4 units of fresh frozen plasma. The patient remained hemodynamically stable and hence was managed conservatively over the next few days. Jaundice which was absent initially developed over the next 2 days with Sr. bilirubin rising to $3.41 \mathrm{mg} / \mathrm{dl}$ on post admission Day(PAD)-2, (Direct- $1.76 \mathrm{mg} / \mathrm{dl}$ and indirect $1.65 \mathrm{mg} / \mathrm{dl}$ ) and $3.86 \mathrm{mg} / \mathrm{dl}$ on PAD-4. (Direct- $1.62 \mathrm{mg} / \mathrm{dl}$, Indirect $-2.24 \mathrm{mg} / \mathrm{dl}$ ) while the liver enzymes improved and became normal by PAD- 5 .
On PAD-10, the patient complained of heaviness of abdomen with distension and on examination the abdomen was tense and tender. On needle aspiration, contents were bile stained and so an abdominal drain was placed under local anesthesia and around 1-1.5Litres of bile stained fluid was drained. Repeat CECT abdomen was done which revealed partial resolution of the earlier lesion with altered morphology of the associated IHBR and left portal vein (Figure 2).
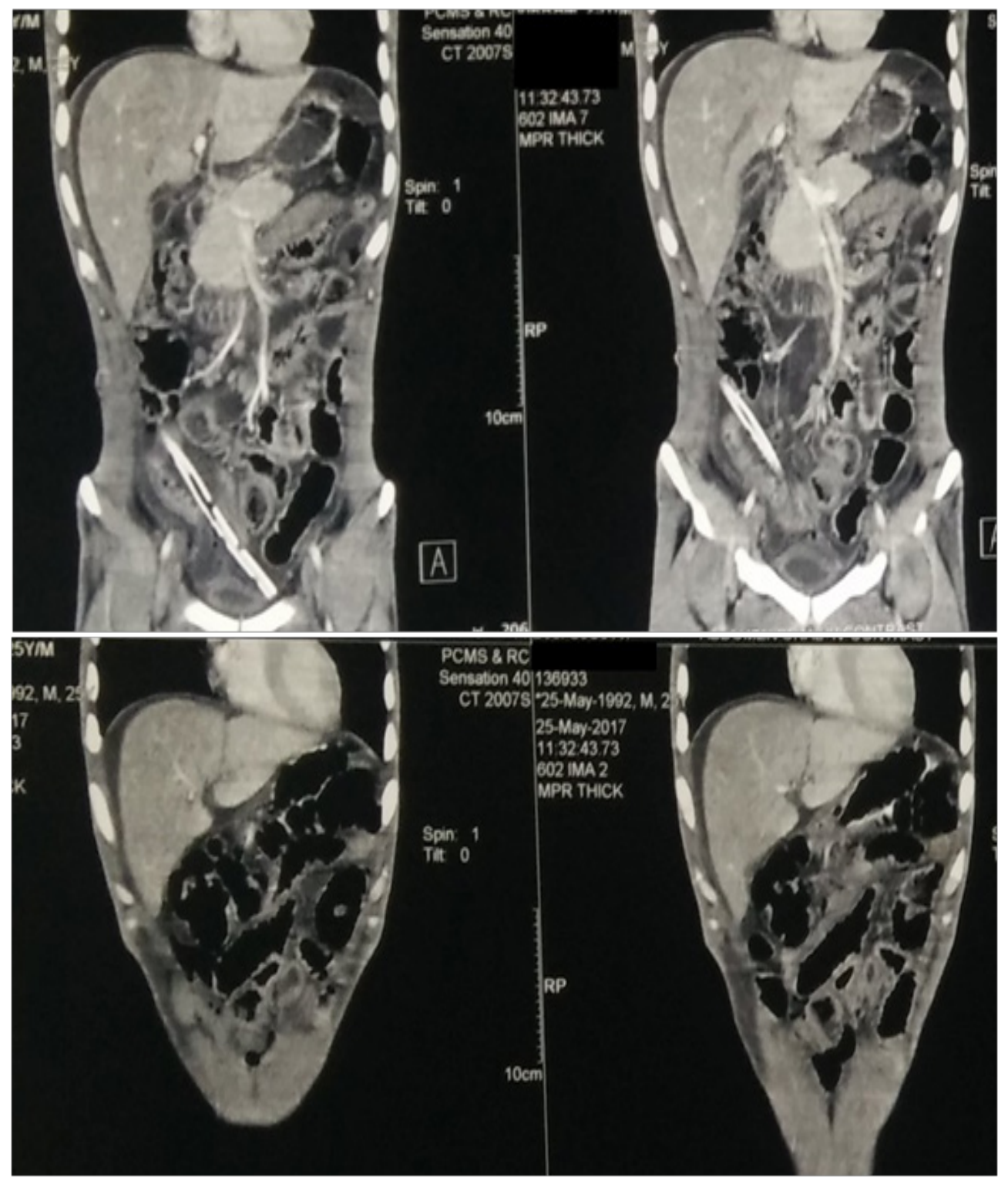

Figure 2 Repeat CECT abdomen showing resolution of the previous lesion with altered morphology of IBHR and Left portal Vein.

The first 24-hourly output in the abdominal drain was around $800 \mathrm{ml}$ and persisted between $500-1000 \mathrm{ml}$ over the next 4days and hence the patient was planned for ERCP.ERCP was done which revealed a leak in proximal CBD for which stenting was done with a DPT plastic stent of $7 \mathrm{FR} * 10 \mathrm{~cm}$ placed across leak. The drain output reduced to less than $100 \mathrm{ml}$ the following day after ERCP and negligible amount thereafter. The following period was un-eventful and the patient was operated for facial injuries and discharged thereafter. He remains in regular follow-up and has been doing well (Figure 3). 


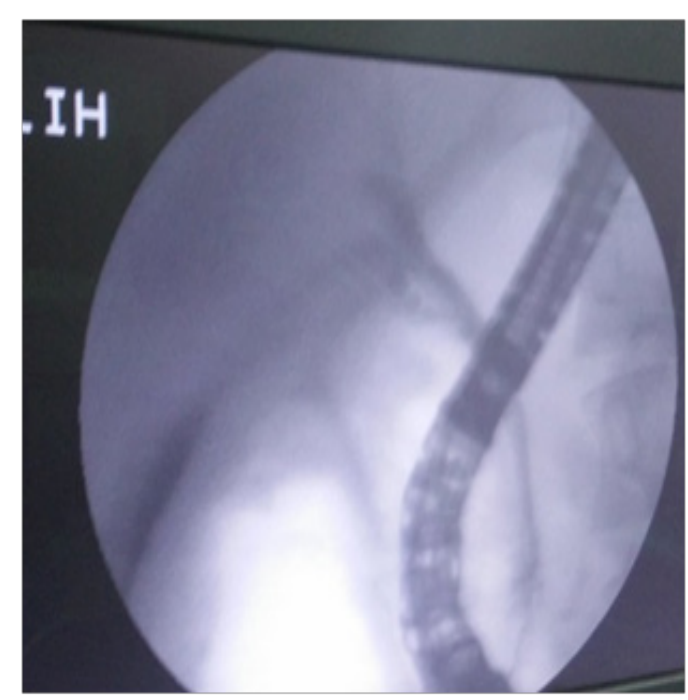

Figure 3 ERCP image showing Leak from the Proximal CBD.

\section{Discussion}

Traumatic injuries of the extrahepatic biliary tract are uncommon, with incidence ranging from 2 to $5 \%$ in abdominal trauma, mainly affecting the gallbladder. ${ }^{2,3,5,6}$ Most lesions of extrahepatic bile ducts are caused by penetrating trauma ${ }^{2,3,7}$ while IBHR injury usually results from blunt trauma with liver involvement. The exact mechanism of injury to the bile duct after blunt trauma is not well known, but a number of factors are identified as responsible for it: (1) a shear force or stretch or avulsion that intersects the duct at its point of attachment to the pancreas or liver; (2) a short cystic duct, causing rapid emptying of the bladder when force is applied, with a sudden increase in intraductal and subsequent predisposition to injury; (3) a shear force applied to the common duct where it is already strained; (4) a compression of the ductal system against the spinal column. ${ }^{1,3,4}$ Perhaps a combination of these factors promotes injury of the extrahepatic biliary system. The most frequent sites of injury to the extrahepatic bile ducts in blunt abdominal trauma are at the upper edge of the pancreas, the hepatic duct bifurcation, and in the origin of the left hepatic duct. $5,8,9$

The clinical presentation virtually divides patients with injuries in the extrahepatic bile ducts into two groups: one with early diagnosis, where laparotomy is indicated because of the presence of hypovolemic shock and signs of peritoneal irritation or associated injuries. The second group is composed of patients with a delayed diagnosis, which are often presented with nausea, vomiting, jaundice, and abdominal pain with distension, as the case in our patient. ${ }^{3,4,10}$

Ultrasonography and computed tomography can result in false negative. Although US is an operator-dependent exam, abdominal ultrasonography was performed twice by different radiologists in the and no injury concerning the biliary tract was observed. The computed tomography common findings in bile duct injury are swelling in the hepatoduodenal ligament, free fluid in the peritoneal cavity, and associated injuries to the liver and duodenum.

Earlier reports showed that the optimal time period from injury to repeat imaging studies for high-grade liver injuries ranges from 7 to 10 days; the mean time for complications to surface. Most bile leaks are diagnosed when a CT scan shows a collection or intra-abdominal fluid. ${ }^{11}$ Although, the presence of free fluid is sensitive, it is non- specific for bile leak. In a recent study of liver lacerated patients, CT scanning showed $98 \%$ hemoperitoneum but only $25 \%$ had bile leak. ${ }^{12}$

If there is no indication for early surgical treatment, the diagnosis of injuries to the extrahepatic bile duct may be delayed, as what had happened in the case ${ }^{3-5}$ Patients not operated on, early in time, and with a biliary fistula may remain asymptomatic for days, because the bile is a sterile component and can be well tolerated. Patients may present few symptoms including abdominal discomfort, nausea, vomiting, jaundice, ascites, and fever. ${ }^{13,14}$ The presence of jaundice after blunt abdominal trauma is suggestive of a missed bile duct injury, but other common diagnoses should be also considered such as massive blood transfusion, liver disease, hepatic trauma, hematoma absorption, or cholecystitis.

Endoscopic retrograde cholangiopancreatography (ERCP) can diagnose and eventually treat bile duct injury with stent even if surgical procedures have been conducted before. ${ }^{13-15}$ The surgical treatment of injuries to the bile ducts should be individualized, based upon hemodynamic stability, associated injuries, and upon the location and extent of the injury. Complications such as biliary fistulae, abscess, and stenosis may be reduced with early diagnosis and appropriate management during surgery. The high morbidity and mortality of these patients are related to associated injuries and their complications.

\section{Acknowledgements}

The author declares there is no conflict of interest

\section{Conflict of interest}

None.

\section{References}

1. Zago TM, Pereira BMT, Calderan TRA, et al. Extrahepatic Duct Injury in Blunt Trauma: Two Case Reports and a Literature Review. Indian J Surg. 2014;76(4):303-307.

2. Brenneman FD, Rizoli SB, Boulanger BR, et al. Blunt left hepatic duct injury. Injury. 1994;25(7):465-467.

3. Ivatury RR, Rohman M, Nallathambi M, et al. The morbidity of injuries of the extra-hepatic biliary system. J Trauma. 1985;25(10):967-973.

4. Melton SM, McGwin G, Cross JM, et al. Common bile duct transection in blunt abdominal trauma: case report emphasizing mechanism of injury and therapeutic management. J Trauma. 2003;54(4):781-785.

5. Nathan M, Gates J, Ferzoco SJ. Hepatic duct confluence injury in blunt abdominal trauma: case report and synopsis on management. Surg Laparosc Endosc Percutan Tech. 2003;13(5):350-352.

6. Feliciano DV, Bitondo CG, Burch JM, et al. Management of traumatic injuries to the extrahepatic biliary ducts. Am J Surg. 1985;150(6):705-709.

7. Gerndt SJ, Seidel SP, Taheri PA, et al. Biliary tract injury following blunt abdominal trauma: case reports. J Trauma. 1995;39(3):612-615.

8. Balzarotti R, Cimbanassi S, Chiara $\mathrm{O}$, et al. Isolated extrahepatic bile duct rupture: a rare consequence of blunt abdominal trauma. case report and review of the literature. World J Emerg Surg. 2012;7(1):16.

9. Posner MC, Moore EE. Extrahepatic biliary tract injury: operative management plan. J Trauma. 1985;25(9):833-837.

10. Ramia JM, Gutiérrez G, Garrote D, et al. Isolated extrahepatic bile duct rupture in blunt abdominal trauma. Am J Emerg Med. 2005;23(2):231232 . 
11. Cuff RF, Cogbill TH, Lambert PJ. Nonoperative management of blunt liver trauma the value of follow-up abdominal computed tomography scans. Am Surg. 2000;66(3):332-336.

12. LeBedis CA, Anderson SW, Mercier G, et al. The utility of CT for predicting umabile leaks in hepatic trauma. Emerg Radiol. 2015;22(2):101107.

13. Bourque MD, Spigland N, Bensoussan AL, et al. Isolated complete tran- section of the common bile duct due to blunt trauma in a child, and review of the literature. J Ped Surg. 1989;24(10):1068-1070.

14. Jones KB, Thomas E. Traumatic rupture of the hepatic duct demonstrated by endoscopic retrograde cholangiography. J Trauma. 1985;25(5):448449.

15. Bade PG, Thomson SR, Hirshberg A, et al. Surgical options in traumatic injury to the extrahepatic biliary tract. Br J Surg. 1989;76(3):256-258. 